\title{
Determination of Tricuspid Regurgitation Velocity/Pulmonary Artery Flow Velocity Time Integral in Dogs with Pulmonary Hypertension
}

\author{
Seungji Kim, Dayoung Oh, Siheon Lee, Sungkyun Hong, Mincheol Choi and Junghee Yoon ${ }^{1}$ \\ College of Veterinary Medicine and the Research Institute for Veterinary Science, Seoul National University, Seoul 08826, Korea
}

(Received: May 27, 2020 / Accepted: August 11, 2020)

\begin{abstract}
This retrospective, echocardiographic study using 144 dogs with clear systolic tricuspid regurgitation on Doppler echocardiography was performed to determine the diagnostic value of the systolic tricuspid regurgitation velocity/pulmonary artery flow velocity time integral to predict the Doppler estimates of dogs with tricuspid regurgitation pressure gradient compared with other cardiac indices of pulmonary hypertension, and to investigate a cutoff value to select patients with a potentially poor outcome. The systolic tricuspid regurgitation velocity/pulmonary artery flow velocity time integral increased significantly as the severity of pulmonary hypertension increased and had a correlation coefficient that was analogous to those of other conventional cardiac indices. A cutoff value greater 1.65 provided the best-balanced sensitivity (84\%) and specificity $(80 \%)$ in determining patients with a poor prognosis. In conclusion, the systolic tricuspid regurgitation velocity/pulmonary artery flow velocity time integral is readily obtained using routine echocardiography and could provide a non-invasive, novel, and supplementary index for evaluating dogs with pulmonary hypertension as useful prognostic criteria, particularly in those with advanced pulmonary hypertension.
\end{abstract}

Key words : dog, echocardiography, pulmonary artery flow velocity time integral, tricuspid regurgitation velocity.

\section{Introduction}

Pulmonary hypertension (PH) is a hemodynamic state in which the pressure of the pulmonary arterial vasculature is elevated $(14,15,26)$. It is a complex syndrome that is caused by a wide range of primary cardiopulmonary and systemic etiologies $(14,26)$. Dogs with PH show variable clinical signs, such as syncope, cough, dyspnea, exercise intolerance, and right-sided congestive heart failure (RCHF) (14). In dogs with myxomatous mitral valve disease (MMVD), concurrent with $\mathrm{PH}$ patients showed poorer prognosis (7). Because of debilitating clinical symptoms and the negative prognostic influence of $\mathrm{PH}$, a prompt and accurate diagnosis is required $(7,15)$. Right-heart catheterization is an accepted gold standard technique for diagnosing PH $(8,10)$. Pulmonary arterial pressure (PAP), pulmonary capillary wedge pressure, and cardiac output $(\mathrm{CO})$ can be measured with right-heart catheterization to determine hemodynamic information regarding the presence and degree of PH (13). However, right-heart catheterization has less clinical value in veterinary medicine because of its invasiveness, cost, and the need for sedation or anesthesia, which can influence $\mathrm{CO}$ and measured pressures (21). Alternatively, Doppler echocardiographic measurement is widely used for the clinical diagnosis $(14,15)$.

Indirect echocardiographic indices for $\mathrm{PH}$, including the tricuspid regurgitation pressure gradient (TRPG), acceleration time of pulmonary artery flow (AT), AT: ejection time (ET), main pulmonary artery diameter:aorta diameter ratio

${ }^{1}$ Corresponding author.

E-mail : heeyoon@snu.ac.kr
(MPA:Ao), right pulmonary artery distensibility index (RPAD index), and other indices of right ventricular function have been proposed. While some studies showed that there was a very good correlation between these indices and catheterization-derived parameters, others implied that these indices had clinical, technical and alignmental limitations (15,23-25,30).

Pulmonary vascular resistance (PVR) (17-19) and pulmonary arterial compliance $(\mathrm{PCa})$ are parameters that are associated with PH (13). The exact value of PVR is calculated invasively as transpulmonary pressure gradient divided by CO $(13,18)$. A definition of PCa is capacity of supporting pulmonary pressure and is determined with the volume of single cardiac stroke. In human medicine, $\mathrm{PCa}$, either alone or combined with PVR, gives clinicians a good stratification of the prognosis of $\mathrm{PH}$ or heart failure (13).

The pulmonary artery flow velocity time integral $\left(\mathrm{VTI}_{\mathrm{PA}}\right)$ correlates well with PVR in human studies (17-19). In addition, the systolic tricuspid regurgitation velocity (TRV)/VTI $\mathrm{PA}_{\mathrm{PA}}$ had a better correlation coefficient with PVR compared with that of $\mathrm{VTI}_{P A}$ alone (18). More recently, $\mathrm{VTI}_{\mathrm{PA}}$ was found to be strongly correlated with $\mathrm{PCa}$, highlighting the need to further investigate the prognostic value and effect of therapy $(5,13)$. However, the reliability and potential diagnostic value of $\mathrm{VTI}_{\mathrm{PA}}$ measurements have not been evaluated in dogs.

Given that PVR and PCa correlated well with the TRV/ $\mathrm{VTI}_{\mathrm{PA}}$ in human medicine studies, we assumed that the TRV/ $\mathrm{VTI}_{\mathrm{PA}}$ could be used as a novel index in dogs with PH and heart failure. We hypothesized that the TRV/VTI $\mathrm{PA}_{\mathrm{PA}}$ may predict the Doppler estimates of PAP, and discriminate between the early and advanced stages of PH in those with RCHF. Therefore, we determined the diagnostic value of TRV/VTI ${ }_{\mathrm{PA}}$ to predict the Doppler estimates of dogs with TRPG com- 
pared with other cardiac indices of PH. Furthermore, a cutoff value was investigated to select patients with a potentially poor outcome.

\section{Materials and Methods}

\section{Animals}

This retrospective study included 144 dogs that had clear TRV on Doppler echocardiography between August 2012 and August 2017. The dogs were divided into four groups based on the echocardiography estimated TRPG that was derived from the TRV. A simplified Bernoulli equation was used: $\mathrm{TRPG}=4 \times(\mathrm{TRV})^{2}$. The groups were: 1$)$ the normal group; dogs with a TRPG $<36 \mathrm{mmHg}, 2$ ) the mild $\mathrm{PH}$ group; dogs with a TRPG $36-50 \mathrm{mmHg}, 3$ ) the moderate $\mathrm{PH}$ group; dogs with a TRPG $50-75 \mathrm{mmHg}$ and 4) the severe PH group; dogs with a TRPG $>75 \mathrm{mmHg}(29,30)$. Exclusion criteria were right ventricular outflow tract obstruction, tricuspid valve dysplasia, ventricular septal defect, cardiomyopathy, and history of taking sildenafil and other vasodilator such as amlodipine, hydralazine and nitroprusside. None of dogs showed evidence of dehydration and those undergoing fluid therapy were excluded from this study because of the possibility that fluid therapy would result in hemodynamic alterations. In the poor prognosis group, we included dogs that showed any signs of RCHF, such as ascites, pleural effusion, and hepatic congestion. Furthermore, the dogs in this group were compared with those without RCHF with a TRPG more than $36 \mathrm{mmHg}$, in whom at least mild $\mathrm{PH}$ was suggested to be present.

\section{Echocardiographic measurements}

Doppler echocardiography studies were performed with an ultrasound unit (Aloka ProSound $\alpha 7$, Hitachi Aloka Medical Ltd., Tokyo, Japan) using a 3-8 MHz phased array sector transducer. For echocardiographic examination, the dogs were manually restrained in the right and left lateral recumbent position. The flow profile of the pulmonary artery with pulse-wave Doppler in the parasternal short axis view, and care was taken to optimize the MPA. The TRV and $\mathrm{VTI}_{\mathrm{PA}}$ was obtained with continuous-wave Doppler in the left apical view (Fig 1). Care was taken to align the sample volume and axis of the blood stream correctly to obtain the highest and clearest Doppler velocity. Five cardiac cycles were recorded for each index. All data were stored on a separate workstation (Infinitt cardiology PACS, Infinitt Healthcare Co., Ltd., Seoul, Korea) to allow for off-line analysis. The following variables were measured: TRV $(\mathrm{m} / \mathrm{s}), \mathrm{VTI}_{\mathrm{PA}}(\mathrm{cm}), \mathrm{RPAD}$ index (\%), AT (ms), AT:ET and MPA:Ao.

\section{Statistical analysis}

All echocardiographic measurements and calculations were performed by the same individual (SJK). An additional observer (DYO) assessed the reliability of the interobserver measurement. The value of the intraclass correlation coefficient (ICC) was interpreted as follows: over 0.75 was excellent, from 0.40 to 0.75 was fair to good, and less than 0.40 was poor (12).

All data were analyzed using statistical analysis software (SPSS version 23.0 for Windows, SPSS Inc., Chicago, IL, USA). Data are described as the mean and standard deviation. A $P$ value of $<0.05$ was considered statistically significant.

Normality was determined with the Shapiro-Wilk test. Analysis of variance was used to evaluate difference among groups; subsequently a post hoc test was performed with Bonferroni's or Dunnett's test. The Kruskal Wallis test was used if the data did not show normality. The JonckheereTerpstra test was used to identify trends among groups. To predict the TRPG, using the cardiac indices, Pearson's correlation and a simple linear regression analysis were used. The Mann-Whitney's test was performed to identify whether the indices could predict poor prognosis. A receiver operating characteristic (ROC) curve was used to suggest a cutoff value, sensitivity, and specificity.

\section{Results}

\section{Clinical and echocardiographic data}

The study population consisted of 144 dogs that were, allocated into four groups. There were no significant differences in body weight, body surface area (BSA), and age among the dogs in the four groups. There were 41 Maltese, 35 Shih Tzu, 14 Miniature Schnauzers, 11 Pekingese, 10 Cocker Spaniels, 8 mixed breeds, 7 Miniature Poodles, 6 Yorkshire Terriers, 3 Pomeranian, 2 Jindo, 2 Chihuahuas, 1 Dachshund, 1 French bulldog, 1 Miniature Pinscher, 1 Pug and 1 Cavalier King
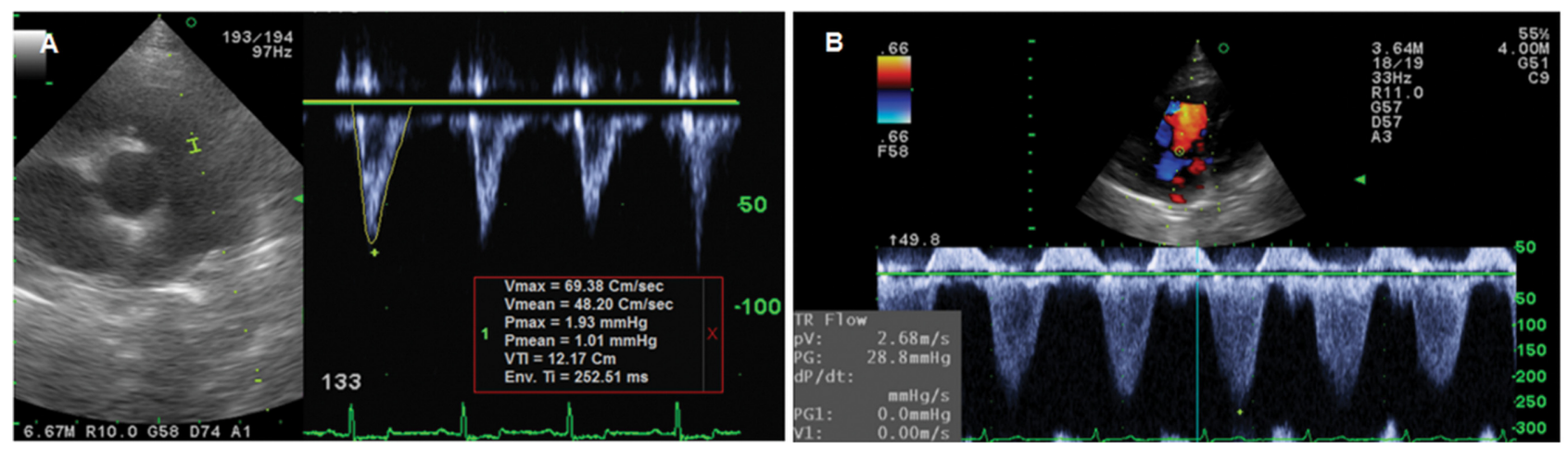

Fig 1. Images of $\operatorname{VTI}_{\mathrm{PA}}(\mathrm{A})$ and $\mathrm{TRV}(\mathrm{B})$ in a $\operatorname{dog}\left(\mathrm{VTI}_{\mathrm{PA}}: 12.17 \mathrm{~cm}\right.$, peak systolic TRV: $\left.2.68 \mathrm{~m} / \mathrm{s}\right)$. VTI $\mathrm{PA}_{\mathrm{PA}}$, pulmonary artery flow velocity time integral; TRV, tricuspid regurgitation velocity. 
Table 1. The clinical and echocardiographic characteristics of the dogs in this study $(n=144)$

\begin{tabular}{lcccc}
\hline \hline Data & Normal $(\mathrm{n}=41)$ & Mild PH $(\mathrm{n}=39)$ & Moderate PH $(\mathrm{n}=32)$ & Severe PH $(\mathrm{n}=32)$ \\
\hline Body weight $(\mathrm{kg})$ & $5.2 \pm 2.7$ & $6.5 \pm 3.7$ & $5.0 \pm 2.7$ & $4.9 \pm 1.9$ \\
BSA $\left(\mathrm{m}^{2}\right)$ & $0.294 \pm 0.093$ & $0.337 \pm 0.120$ & $0.284 \pm 0.103$ & $0.283 \pm 0.075$ \\
Heart rate (bpm) & $128 \pm 30$ & $124 \pm 26$ & $142 \pm 32$ & $153 \pm 32^{\mathrm{a}, \mathrm{b}}$ \\
Age (years) & $12.1 \pm 2.1$ & $12.4 \pm 2.4$ & $12.7 \pm 3.1$ & $11.4 \pm 2.8$ \\
TRPG (mmHg) & $29.8 \pm 4.0$ & $41.2 \pm 3.6^{\mathrm{a}}$ & $62.3 \pm 7.2^{\mathrm{a}, \mathrm{b}}$ & $94.3 \pm 22.3^{\mathrm{a}, \mathrm{b}, \mathrm{c}}$ \\
VTI $_{\mathrm{PA}} /$ BSA & $45.4 \pm 13.5$ & $41.1 \pm 12.2$ & $37.4 \pm 11.5^{\mathrm{a}}$ & $32.2 \pm 11.6^{\mathrm{a}, \mathrm{b}}$ \\
TRV/VTI $_{\mathrm{PA}}$ )/BSA & $0.86 \pm 0.35$ & $0.88 \pm 0.39$ & $1.66 \pm 1.06^{\mathrm{a}, \mathrm{b}}$ & $2.32 \pm 1.08^{\mathrm{a}, \mathrm{b}, \mathrm{c}}$ \\
RPAD index (\%) & $32.6 \pm 7.3$ & $26.7 \pm 6.1^{\mathrm{a}}$ & $22.2 \pm 7.6^{\mathrm{a}, \mathrm{b}}$ & $15.0 \pm 6.5^{\mathrm{a}, \mathrm{b}, \mathrm{c}}$ \\
AT & $67.6 \pm 18.3$ & $66.9 \pm 14.1$ & $53.3 \pm 13.0^{\mathrm{a}, \mathrm{b}}$ & $49.2 \pm 13.4^{\mathrm{a}, \mathrm{b}}$ \\
AT:ET & $0.39 \pm 0.10$ & $0.38 \pm 0.07$ & $0.32 \pm 0.05^{\mathrm{a}, \mathrm{b}}$ & $0.32 \pm 0.08^{\mathrm{a}, \mathrm{b}}$ \\
MPA:Ao & $1.10 \pm 0.10$ & $1.09 \pm 0.11$ & $1.18 \pm 0.14$ & $1.27 \pm 0.19^{\mathrm{a}, \mathrm{b}, \mathrm{c}}$ \\
RCHF (number) & 0 & 0 & 5 & $14^{\mathrm{a}, \mathrm{b}}$
\end{tabular}

Data represented as mean \pm standard deviation. PH, pulmonary hypertension; BSA, body surface area; TRPG, peak tricuspid regurgitation systolic pressure gradient; $\mathrm{VTI}_{\mathrm{PA}}$, pulmonary artery flow velocity time integral; TRV, tricuspid regurgitation velocity; RPAD, right pulmonary artery distensibility; AT, acceleration time of peak pulmonary artery flow; ET, ejection time of pulmonary artery flow; MPA, main pulmonary artery; Ao, aorta; RCHF, right-sided congestive heart failure.

${ }^{\mathrm{a}} P<0.05$ when compared with the normal group. ${ }^{\mathrm{b}} P<0.05$ when compared with the mild $\mathrm{PH}$ group. ${ }^{\mathrm{c}} P<0.05$ when compared with the moderate PH group.

Charles Spaniel. Dogs in the severe PH group had a higher heart rate than did those in the normal and mild $\mathrm{PH}$ groups. A significantly higher number of dogs in the severe $\mathrm{PH}$ group had RCHF $(P<0.05)$ than did those in other groups.

All velocity time integral (VTI) measurements were adjusted by BSA (18). The echocardiographic data of the groups are presented in Table 1.

In the Jonckheere-Terpstra test, TRV/VTI $\mathrm{PA}_{\mathrm{PA}}$ and MPA:Ao were increased and the $\mathrm{VTI}_{\mathrm{PA}}$, RPAD index, AT, and AT:ET were decreased as the severity of PH increased $(P<0.001)$.

\section{Correlation between echocardiographic measurements} and TRPG

The TRV/VTI ${ }_{\mathrm{PA}}$ had correlation coefficient $(R=0.48)$ that was analogous to that of other conventional cardiac indices for predicting TRPG. This parameter had lower correlation coefficient than did the RPAD index, but this parameter's correlation coefficient was higher than AT, AT:ET, and MPA:Ao (Table 2).

To calculate the TRPG non-invasively, a simplified equation, which was derived from TRV/VTI $\mathrm{PA}_{\mathrm{PA}}$ and $\mathrm{VTI}_{\mathrm{PA}}$ was:

$$
\begin{aligned}
& \mathrm{TRPG}=\left(18.895 \times \mathrm{TRV} / \mathrm{VTI}_{\mathrm{PA}}\right)+28.512 \\
& \mathrm{TRPG}=\left(-0.764 \times \mathrm{VTI}_{\mathrm{PA}}\right)+84.569
\end{aligned}
$$

Table 2. Results of Pearson's correlation and simple linear regression analysis of the echocardiographic indices and TRPG

\begin{tabular}{lrcc}
\hline \hline Variable & \multicolumn{1}{c}{$R$} & $R^{2}$ & $P$ \\
\hline VTI $_{\mathrm{PA}} / \mathrm{BSA}$ & -0.37 & 0.14 & $<0.001$ \\
$\left(\mathrm{TRV} / \mathrm{VTI}_{\mathrm{PA}}\right) / \mathrm{BSA}$ & 0.48 & 0.23 & $<0.001$ \\
RPAD index & -0.68 & 0.47 & $<0.001$ \\
AT & -0.47 & 0.22 & $<0.001$ \\
AT:ET & -0.39 & 0.15 & $<0.001$ \\
MPA:Ao & 0.32 & 0.10 & $<0.001$ \\
\hline
\end{tabular}

$R$, correlation coefficient; $R^{2}$, determination coefficient.

\section{ROC curve to identify patients with poor prognosis}

As $\mathrm{PH}$ progressed, mechanical consequences are marked increase in right ventricular afterload, resulting in RCHF. In this study, dogs in the RCHF group had a significantly low median survival time (106 days) and high mortality (86.7\%) compared with dogs without RCHF in the PH group (173 days and $45.2 \%$, respectively).

In dogs in the RCHF group, the TRV/VTI $\mathrm{PA}_{\mathrm{PA}}$ was significantly increased $(P<0.001)$ and the RPAD index $(P=0.033)$ was significantly decreased compared with those in dogs in

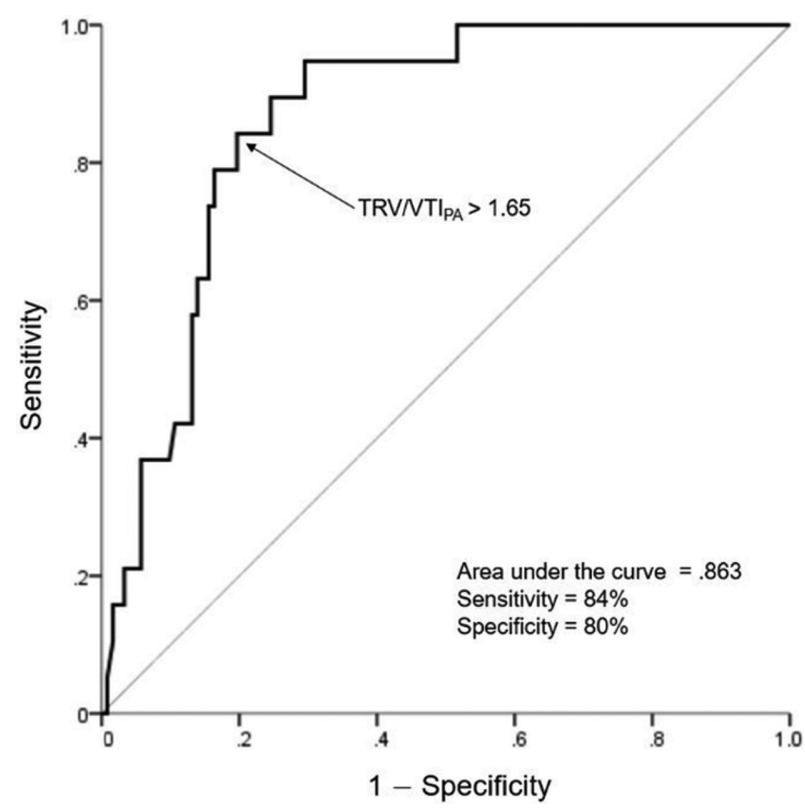

Fig 2. The ROC curve. A TRV/VTI $I_{\mathrm{PA}}$ value of 1.65 provided a sensitivity of $84 \%$ and specificity of $80 \%$ (area under the curve: $0.863,95 \%$ confidence interval: 0.794 to 0.932 ) to identify patients with a poor prognosis. ROC, receiver operating characteristic curve; TRV/VTI $\mathrm{PA}_{\mathrm{PA}}$, systolic tricuspid regurgitation velocity/pulmonary artery flow velocity time integral. 
the $\mathrm{PH}$ group. Using the ROC analysis, we found that a $\mathrm{TRV} / \mathrm{VTI}_{\mathrm{PA}}$ cutoff value of 1.65 provided the best-balanced sensitivity (84\%) and specificity $(80 \%)$ to determine patients with poor prognosis (area under the curve: 0.863). However, the RPAD index did not have clinical value to predict the patient's prognosis (area under the curve: 0.224) (Fig 2).

\section{Intraobserver and interobserver reliabilities}

In the intra- and interobserver agreement analysis, the ICCs of $\operatorname{VTI}_{\mathrm{PA}}$ were excellent (ICCs $>0.9, P<0.001$ ).

\section{Discussion}

In this study, we observed that $\mathrm{TRV} / \mathrm{VTI}_{\mathrm{PA}}$ increased with the severity of $\mathrm{PH}$, and there was an analogous, linear correlation between the TRPG and Doppler-estimated TRV/ $\mathrm{VTI}_{\mathrm{PA}}$ or $\mathrm{VTI}_{\mathrm{PA}}$ compared with other cardiac indices of $\mathrm{PH}$. Additionally, a TRV/VTI $\mathrm{PA}_{\mathrm{PA}}$ over 1.65 , with a sensitivity of $84 \%$ and specificity of $80 \%$, suggested that dogs with $\mathrm{PH}$ had poor prognosis. No significant variation of $\mathrm{VTI}_{\mathrm{PA}}$ was identified in measurement reliability.

Various echocardiographic indices have been used routinely to determine $\mathrm{PH}$; however, such indices have some pitfalls. Heart rate has a significant effect on the ventricular systolic time interval (AT and AT:ET) in dogs $(1,20)$. However, another study indicated that age and heart rate have only negligible effects on these indices (23). Echocardiographic evidence of heart remodeling such as septal flattening or increased MPA:Ao may be observed in dogs with moderate to severe $\mathrm{PH}$, but these changes could be undetected in dogs with in mild PH. The limitation of TRPG, which is used routinely to estimate PAP, occurs especially when evaluating patients who receive vasodilators. Sildenafil is frequently used to treat PH in dogs to decrease PVR; the consequences include increasing $\mathrm{CO}$, whereas the PAP does not change significantly. Therefore, assessing the TRPG alone could underestimate the hemodynamic changes of vasodilators unless the patient remain untreated (18).

An accurate evaluation of pulmonary circulation requires an understanding of both its static and dynamic components. In human medicine, PVR and PCa are hemodynamic concepts that are frequently used in clinical practice. Evaluation of PVR is used to identify right ventricular afterload, but it has limitation as a static component. Reduced PCa is known to be a powerful marker of poor prognosis in patients with idiopathic pulmonary arterial hypertension and those with heart failure. Moreover, studies suggest that $\mathrm{PH}$ could be better diagnosed in the early stage through alteration of $\mathrm{PCa}$, rather than increase in PVR (13). However, measuring these hemodynamic components in vivo, especially in veterinary medicine, is difficult due to its invasiveness and the patient's need for additional sedatives or anesthetics. As alternatives, VTI is available by means of non-invasive parameter. $\mathrm{VTI}_{\mathrm{PA}}$ was first introduced in veterinary medicine, as an index that is measured with Doppler echocardiography in a study about independent factors such as body position, sedation, and exercise that can influence echocardiographic indices (21). However, the diagnostic significance of $\mathrm{VTI}_{\mathrm{PA}}$ has not been evaluated in dogs so far.
Many studies have demonstrated that there is a correlation

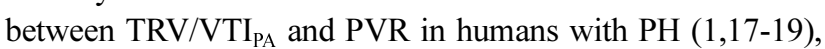
and suggested normal value by age in pediatrics (17). Moreover, evaluating the $\mathrm{VTI}_{\mathrm{PA}}$ in heart failure patients has been shown to be feasible (5). VTI is closely related to CO; the consequences of low $\mathrm{CO}$ are cardiogenic shock, multi-organ dysfunction, and even death (2). Moreover, a low VTI in either the right or left ventricular outflow tract correlates with adverse clinical outcomes $(18,27)$. The rapid ultrasound in shock (RUSH examination) using VTI is an emerging protocol to identify the source of shock or hypotension and predict the patient's response to treatment in the emergency department (6).

The progression of postcapillary $\mathrm{PH}$ is slow, a hypertensive left ventricle adversely affects the compliance and filling of the contralateral right ventricle, resulting in a cycle of vicious impact on function in the two ventricles (9). In veterinary clinics, MMVD that is associated with moderate to severe $\mathrm{PH}$ is also associated with shorter survival times (8). Estimating the TRV/VTI $\mathrm{PA}_{\mathrm{A}}$ may be useful to assess disease over time.

Many equations and cutoff values for screening patients with a high PVR have been suggested through echocardiographic measurement of $\mathrm{TRV} / \mathrm{VTI}_{\mathrm{PA}}$ in diseased humans $(16,19,20,23)$. However, its reliability differs according to various inclusion criteria $(4,27)$. For example, echocardiography was shown to be useful to screen patients with $\mathrm{PH}$ and PVR greater than 2 wood units using TRV/VTI $I_{\mathrm{PA}}$ value of 0.14 as the cutoff. However, the authors of that study concluded that this cutoff value was disappointing for the accurate assessment of higher PVR (22). Alternatively, another study suggested a linear regression equation that was applicable to patients who had severe PH with a PVR greater than 6 wood units (3). Because the pulmonary pressure in our dogs ranged broadly and the hemodynamic values could not be measured directly, the linear regression equation in this study needs further validation. There is an absence of studies about a normal VTI value that is based on age, body weight, BSA, heart rate, and various cardiopulmonary diseases, further studies are needed to evaluate healthy and ill dogs.

In our study, group of poor prognosis were confined to dogs with RCHF. Unlike in humans, identifying RCHF in dogs depends entirely on the evidence that is seen on radiography or ultrasonography, such as ascites, pleural effusion, and pericardial effusion. Therefore, the cutoff value that was suggested in this study could be overestimated or inaccurate.

As client-owned dogs were included, indirect measurements of hemodynamic value could be suggested as potential limitations. In this study design, the degree and presence of PH were determined with the TRPG. This method is known to be less accurate than invasively determined PAP $(10,28)$. The accuracy of invasive systolic PAP was minimally improved when adding right atrial pressure to the TRPG (25). Moreover, calculating the right atrial pressure could result in overestimation of the severity of PH (11). For reasons mentioned above, we neglected to study right atrial pressure to estimate the Doppler derived PAP. Many studies that evaluated the relationship between Doppler echocardiography-derived PAP and various cardiac indices showed simi- 
lar results, although slightly different methods were used in each study to estimate PAP.

The authors pursued to evaluate the importance of $\mathrm{VTI}_{\mathrm{PA}}$ and in clinical settings, $\mathrm{VTI}_{\mathrm{PA}}$ could provide additional information on individual hemodynamics as an alternative to invasive PVR or PCa in PH dogs. Moreover, the TRV/VTI $\mathrm{PA}_{\mathrm{PA}}$ cutoff value can propose whether the $\mathrm{PH}$ patients are acceptable or abnormally impaired. It allows for good clinical stratification of the prognosis particularly in progressed PH. In conclusion, it is suggested that $\mathrm{TRV} / \mathrm{VTI}_{\mathrm{PA}}$ could be included as a simple and non-invasive parameter to determine the severity of $\mathrm{PH}$ through routine echocardiographic examination.

\section{References}

1. Abbas AE, Franey LM, Marwick T, Maeder MT, Kaye DM, Vlahos AP, Serra W, Al-Azizi K, Schiller NB, Lester SJ. Noninvasive assessment of pulmonary vascular resistance by Doppler echocardiography. J Am Soc Echocardiogr 2013; 26: 1170-1177.

2. Abraham WT, Adams KF, Fonarow GC, Costanzo MR, Berkowitz RL, LeJemtel TH, Cheng ML, Wynne J. Inhospital mortality in patients with acute decompensated heart failure requiring intravenous vasoactive medications: an analysis from the acute decompensated heart failure National Registry. J Am Coll Cardiol 2005; 46: 57-64.

3. Ajami GH, Cheriki S, Amoozgar H, Borzouee M, Soltani M. Accuracy of Doppler-derived estimation of pulmonary vascular resistance in congenital heart disease: An index of operability. Pediatr Cardiol 2011; 32: 1168-1174.

4. Atkins CE, Snyder PS. Systolic time intervals and their derivatives for evaluation of cardiac function. $J$ Vet Intern Med 1992; 6: 55-63.

5. Bhattacharya PT, Troutman GS, Mao F, Fox AL, Tanna MS, Zamani P, Grandin EW, Menachem JN, Birati EY, Chirinos JA, Mazimba S, Smith KA, Kawut SM, Forfia PR, Vaidya A, Mazurek JA. Right ventricular outflow tract velocity time integral-to-pulmonary artery systolic pressure ratio: a noninvasive metric of pulmonary arterial compliance differs across the spectrum of pulmonary hypertension. Pulm Circ 2019; 9: $1-10$.

6. Blanco P, Aguiar FM, Blaivas M. Rapid ultrasound in shock (RUSH) velocity-time integral: A proposal to expand the RUSH protocol. J Ultrasound Med 2015; 34: 1691-1700.

7. Borgarelli M, Abbott J, Braz-Ruivo D, Chiavegato D, Crosara S, Lamb K, Ljungvall I, Poggi M, Santilli RA, Haggstrom J. Prevalence and prognostic importance of pulmonary hypertension in dogs with myxomatous mitral valve disease. J Vet Intern Med 2015; 29: 569-574.

8. Brecker SJ, Gibbs JS, Fox KM, Yacoub MH, Gibson DG. Comparison of Doppler derived haemodynamic variables and simultaneous high fildelity pressure measurements in severe pulmonary hypertension. Br Heart J 1994; 72: 384-389.

9. Bronicki RA, Baden HP. Pathophysiology of right ventricular failure in pulmonary hypertension. Pediatr Crit Care Med 2010; 11: S15-22.

10. Currie PJ, Seward JB, Chan KL, Fyfe DA, Hagler DJ, Mair DD, Reeder GS, Nishimura RA, Tajik AJ. Continuous wave Doppler determination of right ventricular pressure: a simultaneous Doppler-catheterization study in 127 patients. J Am Coll Cardiol 1985; 6: 750-756.

11. Fisher MR, Forfia PR, Chamera E, Housten-Harris T, Champion HC, Girgis RE, Corretti MC, Hassoun PM. Accuracy of
Doppler echocardiography in the hemodynamic assessment of pulmonary hypertension. Am J Respir Crit Care Med 2009; 179: 615-621.

12. Fleiss JL. Design and analysis of clinical experiments. New York: Wiley. 1999: 23-27.

13. Ghio S, Schirinzi S, Pica, S. Pulmonary arterial compliance: How and why should we measure it? Glob Cardiol Sci Pract. 2015; 4: 58.

14. Johnson L, Boon J, Orton C. Clinical characteristics of 53 dogs with Doppler-derived evidence of pulmonary hypertension: 1992-1996. J Vet Intern Med 1999; 13: 440-447.

15. Kellihan HB, Stepien RL. Pulmonary hypertension in canine degenerative mitral valve disease. J Vet Cardiol 2012; 14: 149-164.

16. Koestenberger M, Avian A, Grangl G, Burmas A, Kurath-Koller S, Hansmann G. Right ventricular outflow tract velocity (RVOT VTI) and tricuspid regurgitation velocity/RVOT VTI ratio in pediatric pulmonary hypertension. Int J Cardiol 2016; 212: 274-276.

17. Koestenberger M, Nage B, Ravekes W, Avian A, Burmas A, Grangl G, Cvirn G, Gamillscheg A. Right ventricular outflow tract velocity time integral determination in 570 healthy children and in 52 pediatric atrial septal defect patients. Pediatr Cardiol 2015; 36: 1129-1134.

18. Kouzu H, Nakatani S, Kyotani S, Kanzaki H, Nakanishi N, Kitakaze M. Kouzu, H., Nakatani, S., Kyotani, S. Noninvasive estimation of pulmonary vascular resistance by Doppler echocardiography in patients with pulmonary arterial hypertension. Am J Cardiol 2009; 103: 872-876.

19. Pande A, Sarkar A, Ahmed I, Naveen Chandra G, Patil SK, Kundu CK, Arora R, Samanta A. Non-invasive estimation of pulmonary vascular resistance in patients of pulmonary hypertension in congenital heart disease with unobstructed pulmonary flow. Ann Pediatr Cardiol 2014; 7: 92-97.

20. Pipers FS, Andrysco RM, Hamlin RL. A totally non-invasive method for obtaining systolic time intervals in the dog. Am J Vet Res 1978; 39: 1822-1826.

21. Rhinehart JD, Schober KE, Scansen BA, Yildiz V, Bonagura JD. Effect of body position, exercise, and sedation on estimation of pulmonary artery pressure in dogs with degenerative atrioventricular valve disease. J Vet Intern Med 2017; 31: 1611-1621

22. Roule V, Labombarda F, Pellissier A, Sabatier R, Lognoné T, Gomes S, Bergot E, Milliez P, Grollier G, Saloux E. Echocardiographic assessment of pulmonary vascular resistance in pulmonary arterial hypertension. Cardiovasc Ultrasound 2010; 8: 21 .

23. Schober KE, Baade H. Doppler echocardiographic prediction of pulmonary hypertension in West Highland White Terriers with chronic pulmonary disease. J Vet Intern Med 2006; 20: 912-920.

24. Serres F, Chetboul V, Gouni V, Tissier R, Sampedrano CC, Pouchelon J. Diagnostic value of echo-Doppler and tissue Doppler imaging in dogs with pulmonary arterial hypertension. J Vet Intern Med 2007; 21: 1280-1289.

25. Soydan LC, Kellihan HB, Bates ML, Stepien RL, Consigny DW, Bellofiore A, Francois CJ, Chesler NC. Accuracy of Doppler echocardiographic estimates of pulmonary artery pressure in a canine model of pulmonary hypertension. J Vet Cardiol 2015; 17: 13-24.

26. Stepien RL. Pulmonary arterial hypertension secondary to chronic left-sided cardiac dysfunction in dogs. J Small Anim Pract 2009; 50S: 34-43.

27. Tan C, Rubenson D, Srivastava A, Mohan R, Smith MR, 
Billick K, Bardarian S, Heywood JT. Left ventricular outflow tract velocity time integral outperforms ejection fraction and Doppler derived cardiac output for predicting outcomes in a select advanced heart failure cohort. Cardiovasc Ultrasound 2017; 15: 18 .

28. Uehara Y. An attempt to estimate the pulmonary artery pressure in dogs by means of pulsed Doppler echocardiography. J Vet Med Sci 1993; 55: 307-312.

29. Vezzosi T, Domenech O, Iacona M, Marchesotti F, Zini E,
Venco L, Tognetti R. Echocardiographic evaluation of the right atrial area index in dogs with pulmonary hypertension. $\mathrm{J}$ Vet Intern Med 2018; 32: 42-47.

30. Visser LC, Im MK, Johnson LR. Diagnostic value of right pulmonary artery distensibility index in dogs with pulmonary hypertension: comparison with Doppler echocardiographic estimates of pulmonary artery pressure. J Vet Intern Med 2016; 30: 543-552. 